\title{
Pensamientos sobre como enfrentar el sufrimiento en personas mentalmente discapacitadas y que padecen una enfermedad grave
}

\author{
Thoughts on dealing with suffering in mentally ill patients affected by a severe disease
}

Margarita Arancibia Silva ${ }^{1}$.

\begin{abstract}
RESUMEN
La práctica de la Medicina pone en nuestro camino constantemente dilemas éticos y morales. Se plantea el enfrentamiento del médico al sufrimiento de pacientes con discapacidad mental y que padecen una enfermedad grave. Se reflexiona sobre la relación médico paciente, el respeto a los principios bioéticos y los conflictos éticos en el tratamiento de estos pacientes en particular.
\end{abstract}

\section{ABSTRACT}

In the course of medical practice, physicians are often confronted with ethical and moral dilemmas. Dealing with suffering in mentally ill patients affected by a severe disease poses one such dilemma. Doctor/patient relationship, respect for bioethical principles, and ethical issues in treating these patients are discussed.

\section{INTRODUCCIÓN}

Se entiende por ética la tendencia natural que tienen los seres humanos para tener en cuenta el bien en sus acciones, siendo esta tendencia natural tan incuestionable, que cuando alguien opta por elegir el mal, se ha llegado a suponer que ha sido víctima de la posesión de su alma por algún espíritu demoníaco ${ }^{1}$. Actualmente en el ámbito psicológico se dice que tiene alguna psicopatía.

Este concepto de tendencia a elegir el bien implica un sentido de generosidad entre unos y otros.

Así el término ética se ha usado para designar filosofía moral o práctica, entendiéndolo como una reflexión filosófica sobre la acción humana, en sus aspectos susceptibles de ser calificados como buenos, correctos o incorrectos, es decir aspectos llamados morales².

La bioética surge como la necesidad de la existencia de una disciplina cuyo objetivo es regular la acción de la ciencia sobre el hombre, como también las acciones del hombre con relación al medio ambiente, el derecho, la política, las comunicaciones, etc. Así mismo constituye en la actualidad una disciplina que ayuda a la solución práctica y contextualizada de los problemas derivados de la confrontación de la tecnociencia y la dignidad y respeto que merece siempre el ser humano ${ }^{3}$.

Actualmente se presentan conflictos éticos en la clínica, en relación a factores como, el avance de las capacidades tecnológicas para prolongar la vida, la búsqueda de una redefinición del rol del

Médico del Servicio de Otorrinolaringología, Hospital San Juan de Dios. Santiago. 
paciente en la toma de decisiones médicas y el reconocimiento del derecho de éste para decidir algunos aspectos del tratamiento médico y el rol del médico en la definición del mejor beneficio de su paciente; la existencia de presiones para reducir los costos de la atención médica cuando los recursos son limitados, que llevan a decidir sobre la base de consideraciones éticas, la mejor asignación de los mismos; la tendencia a hacer pública y eventualmente llevar a la justicia, la discusión de decisiones que tradicionalmente quedaba circunscrita al ámbito médico, el paciente y su familia ${ }^{4}$.

\section{PACIENTES CON DISCAPACIDAD MENTAL}

La práctica médica nos pone reiteradamente en dificultades éticas y morales que no son fáciles de resolver. Muchas veces estamos frente a pacientes que no son capaces de expresar sus sentimientos, lo que desean y sus miedos. Ante eso habitualmente solicitamos a sus familiares que tomen una decisión ante la solicitud del equipo médico.

Cabe en esta circunstancia, pensar si éstos están tomando una decisión en base a lo que ellos mismos querrían si se encontrasen en esta situación, o lo que ellos piensan que su pariente desearía si pudiera decidir el mismo. También puede suceder que estos parientes tomen la decisión considerándose ellos como personas con mayor sabiduría, y creyendo saber lo que mejor le conviene a su enfermo 0 a ellos mismos. No siempre esta decisión es la misma en cada una de estas posibilidades, ya que a veces se puede optar por recuperar el paciente a pesar de todo, sin importar los costos emocionales, personales o económicos, otras veces piden hacer todo lo posible por recuperarlo evitando al máximo su sufrimiento y en otras ocasiones lo que se solicita es limitar el esfuerzo terapéutico dadas las condiciones del paciente.

A veces también nos enfrentamos a que no hay familia que decida y entonces tenemos que tomar la decisión como equipo médico, autorizados por alguna autoridad legal. Aquí también estamos enfrentados a las mismas posibilidades anteriores, además de las propias vicisitudes del deseo del médico y sus sentimientos ante el éxito o fracaso de su decisión.

En los pacientes con discapacidad mental surgen desafíos en la relación médico paciente y en la aceptación del consentimiento informado. Se debe mantener el respeto por los principios bioéticos básicos, y solicitar muchas veces la opinión de un Comité de Ética.

A continuación se plantean parte de estos dilemas.

\section{RELACIÓN MÉDICO PACIENTE}

Por casi 2500 años el Juramento Hipocrático, ha sido el código por el que se han regido los médicos, sin embargo, en las últimas décadas su prestigio en los profesionales médicos y escuelas de medicina ha disminuido. Esto generó un movimiento de las sociedades médicas de Estados Unidos y Europa que reunió un comité en 1999 que se abocó a trabajar en lo que se llamó Carta del Profesionalismo Médico, para desarrollar un nuevo juramento ${ }^{5}$.

Tradicionalmente la relación médico paciente fue del tipo paternalista, basada en que el médico es quien tiene el conocimiento y «sabe» lo que le conviene 0 lo que es mejor para su paciente, sin embargo dado el desarrollo de las sociedades, el acceso a la educación, a las libertades y derechos humanos, se ha producido un cambio en este tipo de relación. Cada vez hay más información médica disponible, no sólo por medios científicos, sino también en periódicos y programas de televisión, a veces más publicitarios que culturales.

Actualmente ha habido un cambio a un modelo informativo, interpretativo $\mathrm{y} / 0$ deliberativo y es el paciente quien en forma cada vez más personal, define si alguien más puede ser informado sobre su estado y decide sobre lo que se le propone, según sus propios valores ${ }^{6}$. Por tanto, un paciente enfrentado a una enfermedad pide y exige a su médico información clara y precisa sobre la naturaleza de lo que padece y cuáles son los riesgos y beneficios de lo ofrecido. Sin embargo en los pacientes que no pueden decidir será la familia 0 su representante quien lo haga.

\section{RESPETAR LOS PRINCIPIOS BIOÉTICOS}

Los principios básicos de la relación médico paciente como son Autonomía, Beneficencia, No-maleficencia 
y Justicia, por lo tanto cobran vital importancia en pacientes limitados en su capacidad de decisión frente al acto médico. En ellos también debe respetarse la intimidad, confidencialidad y la veracidad de esta relación.

La Autonomía ha pasado ha cobrar gran importancia desde que se publicó el Informe Belmont en 19787. En este informe se declara además que «se deben tutelar los derechos de las personas cuya autonomía está disminuida o comprometida», ello nos obliga también a cautelar el principio de Beneficencia y por sobre todo No-maleficencia. Este principio se considera independiente ya que el deber de no dañar es imperativo sobre el de promover el bien del enfermo.

Así pues en ocasiones nos enfrentamos a pacientes que sufren una enfermedad grave en los que no es posible realizar un consentimiento informado y les corresponde a otros determinar «su bien». Este puede ser mejorar su enfermedad y a veces sólo evitar o disminuir su sufrimiento.

Esta decisión no es sencilla y se debe solicitar la ayuda a quienes tengan una mayor experiencia en ello. Aquí es donde se debe solicitar la opinión a los Comités de Ética.

Estos comités nacieron alrededor de 1962 en Seattle a raíz de la creación de la máquina de hemodiálisis, la que inicialmente no estaba disponible masivamente y por ello había que decidir quiénes eran los que se beneficiarían con este nuevo tratamiento, lo que implicaba una discriminación médica. La pregunta por lo tanto era ¿por qué un avance médico debería crear una nueva discriminación médica? ¿Quién y cómo elegía a los candidatos? La novedad estribaba precisamente en que la respuesta a estos interrogantes no recaía sobre los médicos, sino sobre una representación de la comunidad. Posteriormente en 1975, debido al caso Karen Ann Quinlan, el Tribunal Supremo de Nueva Jersey que autorizó su desconexión del respirador artificial, recomendó la creación de estos comités en los hospitales para enfrentar estos conflictos.

\section{COMITÉS DE ÉTICA ASISTENCIAL}

Los Comités de Ética Asistenciales (CEA) nacen entonces como una comisión consultiva e interdisciplinaria, creada para analizar y asesorar en la resolución de posibles conflictos éticos que se producen en la práctica clínica. Solo tienen autoridad moral. Algunas de sus principales funciones son:

1. Proteger los derechos de los pacientes

2. Analizar, asesorar y facilitar el proceso de decisión clínica en las situaciones que creen conflictos éticos entre el personal sanitario, los pacientes o usuarios y las instituciones

3. Colaborar en la formación bioética de los profesionales del hospital y del área de la salud y en particular de los miembros del comité ${ }^{8}$.

Los comités al ser consultados emitirán un informe que deberá quedar en la ficha. Hay algunas recomendaciones para analizar los casos clínicos ${ }^{9,10}$.

Los casos extremos son los que ponen en jaque a los equipos médicos y CEA, especialmente cuando el paciente está incapacitado permanentemente para comprender su estado y no será posible obtener el consentimiento informado, como puede darse en niños menores, pacientes con retado mental, estado vegetativo persistente, etc.

\section{CONFLICTOS ÉTICOS A TENER EN CUENTA}

El consentimiento informado actualmente es imprescindible, sin embargo hay pacientes en los que no es posible obtenerlo. Esto puede ser porque el paciente siendo competente, se encuentra grave 0 porque tiene una discapacidad mental que le impide comprender su condición.

En pacientes no competentes, se debe solicitar un consentimiento subrogado, que deberá ser ejercido por la persona que el paciente haya eventualmente determinado cuando era competente 0 por quien la familia determine. Actualmente en algunos países, se ha legislado sobre voluntades anticipadas, esto es para prever situaciones por las que se quisiera evitar, en caso de enfermedad grave.

La calidad de vida no siempre es objetiva. En ocasiones solo no tener dolor es motivo para dar gracias, en otras ocasiones la pérdida de una función puede hacer sentir al sujeto, que su vida no tiene sentido. Por ello hay que poner todo el empeño en la comunicación con el paciente, para poder ayudarlo en el más amplio sentido y estar dispuestos en ocasiones a no continuar una terapia, es decir limitar el esfuerzo terapéutico, si no 
está ayudando a evitar el sufrimiento. En pacientes sin comprensión la evaluación de la calidad de vida será hecha por sus familiares o el equipo médico.

Cuando la enfermedad es intratable se deberá realizar manejo de los síntomas, evitar el dolor, no realizar manipulaciones médicas innecesarias (procedimiento fútil), y mejorar secuelas que puedan haber quedado. Aquí es de vital importancia los cuidados paliativos. Estos no sólo deben implementarse en el centro hospitalario, sino también en el cuidado domiciliario.

Si se trata de un paciente en Estado Termina ${ }^{11}$ además de los cuidados paliativos, será necesario ayudarlo a bien morir (muerte digna), esto es en su casa si es posible, acompañado de sus seres queridos, con ayuda espiritual si así lo requiere, y ayudarlo a asumir su propia muerte.

\section{CONCLUSIÓN}

Los avances médicos en conocimiento y tecnología han permitido el diagnóstico y tratamiento de muchas patologías que anteriormente eran intratables. Estos avances no solo permiten el tratamiento de estas enfermedades, sino también la prolongación de la vida en pacientes que no pueden ser curados y cuya dolencia es «sufrida» por largo tiempo.

En pacientes con discapacidad mental es la familia y el equipo médico quienes toman decisiones sobre un determinado tratamiento y cuando la enfermedad es grave, muchas veces la decisión es controversial.

El desarrollo de la bioética a nivel hospitalario, nos recuerda que ante estos pacientes igualmente, se debe mantener el respeto a la vida humana en todo ámbito, considerando los principios básicos de Autonomía, Beneficencia, No maleficencia y Justicia, y tener en cuenta especialmente a pacientes con discapacidad mental. En ellos es igualmente importante mantener una relación equipo médico-paciente familia, adecuada.

En estos casos se nos presentan conflictos éticos que debemos resolver y solicitar ayuda a los
Comités de Ética. Cuando la enfermedad es intratable 0 se trata de un enfermo terminal se le deben otorgar cuidados paliativos y se debe procurar una muerte digna junto sus seres queridos tanto en la sala de hospital como en su casa.

\section{BIBLIOGRAFÍA}

1. Roa A. En: Ética y bioética. Edit Andrés BeIlo.1998. 19-25

2. Escríbar A. Bioética: su sentido y su función. Rev Med Clin Condes 14,2:104-6

3. Pérez M. Bioética. Rev Med Clin Condes; 13,4:157-160

4. Lavados M. En: Ética clínica, fundamentos y aplicaciones. Ediciones Universidad Católica de Chile.1993. 19

5. Project of the ABIM Foundation, ACP-ASIM Foundation, and European Federation of Internal Medicine. Medical Professionalism in the New Millennium: A Physician Charter. Ann Int Med 2002; 136:243-6.

6. Emanuel EJ y Emanuel LL. Cuatro modelos de la relación médico-paciente». En: Bioética para clínicos, Cruceiro A. (edit). Ed. Castellana.

7. Cf. informe Belmont, publicado por el Ministerio de Sanidad y Consumo en el volumen Ensayos Clínicos en España (1982-1988), Madrid 1990, anexo 4.

8. Álvarez JC. Comisión de Ética Asistencial. Reflexión sobre sus Funciones y Funcionamiento. En: Bioética: un diálogo plural. Ferrer JJ y Martínez JL (edit). Ed. Universidad Pontificia de Comillas. Madrid, España. 2002: 367-84.

9. TABOADA P. Ética clínica: principios básicos y modelo de análisis. Bol ESC Medicina PUC 1998, 27:7-13.

10. Gracia D. Metodología de deliberación en bioética clínica.

11. Grupo de Estudios de Ética Clínica de la Sociedad Médica de Santiago. El enfermo terminal. Rev Méd Chile 2000; 128: 547-52. 